ఠ

\title{
Diclofenac sodium topical solution with dimethyl sulfoxide, a viable alternative to oral nonsteroidal anti-inflammatories in osteoarthritis: review of current evidence
}

\author{
This article was published in the following Dove Press journal: \\ Journal of Multidisciplinary Healthcare \\ 8 July 2011 \\ Number of times this article has been viewed
}

\author{
Philip Fuller' \\ Sanford Roth ${ }^{2}$ \\ 'Covidien, Hazelwood, MO; ${ }^{2}$ Arizona \\ Research and Education, Arthritis \\ Research Laboratory, Arizona State \\ University, Phoenix, \\ AZ, USA
}

\begin{abstract}
Topical nonsteroidal anti-inflammatory drugs (NSAIDs) may offer a safer alternative to their oral counterparts for the management of osteoarthritis. Diclofenac sodium topical solution with dimethyl sulfoxide (TDiclo) was evaluated in five randomized, controlled trials and is indicated for treatment of the signs and symptoms associated with osteoarthritis of the knee. Three studies showed that TDiclo is superior to placebo and vehicle control with respect to pain, physical function, and perception of osteoarthritis symptoms. Two studies showed that benefits are similar to those of oral diclofenac, with one study demonstrating statistical equivalence. The most common adverse event associated with TDiclo in these studies was dry skin. Incidences of gastrointestinal adverse events and abnormal levels of liver enzymes were lower with TDiclo compared with oral diclofenac in active-controlled studies. Based on these studies, TDiclo represents a practical, evidence-based option for the management of osteoarthritis of the knee.
\end{abstract}

Keywords: osteoarthritis, nonsteroidal anti-inflammatory drugs, diclofenac, topical analgesic

\section{Introduction}

Osteoarthritis is the most common form of arthritic disease. It is estimated that 27 million US adults suffer from osteoarthritis, more than nine million of whom have symptomatic osteoarthritis of the knee. ${ }^{1}$ The prevalence of osteoarthritis is expected to rise as the population ages. Osteoarthritis has a high economic burden, with costs attributable to treatment and the effects of disability and comorbidity, and indirect costs, such as loss of earnings. ${ }^{2}$

Because there are currently no disease-modifying therapies for osteoarthritis, modern management aims to relieve pain and improve function and health-related quality of life..$^{3-8}$ Pharmacological management of osteoarthritis usually begins with acetaminophen and progresses to traditional nonselective nonsteroidal antiinflammatory drugs (NSAIDs) or cyclo-oxygenase-2 (COX-2) selective NSAIDs. Most patients with osteoarthritis are likely to receive oral traditional NSAIDs or COX-2 inhibitors; however, the associated gastrointestinal, renal, and cardiovascular adverse effects of these agents can potentially limit long-term use. ${ }^{9-15}$ Topical formulations of NSAIDs, recently approved by the US Food and Drug Administration (FDA) for the treatment of osteoarthritis, have the potential to offer a safe alternative to oral NSAIDs due to decreased systemic exposure to the active molecule. ${ }^{16}$
Correspondence: Philip Fuller Covidien, 675 McDonnell Blvd, Hazelwood, MO 63042, USA

$\mathrm{Tel}+\mathrm{I} 3 \mid 46543415$

Fax + I 3146543755

Email philip.fuller@covidien.com 
Several international and national guidelines for the management of osteoarthritis are available, ${ }^{3-8}$ all of which recommend a similar, multifaceted approach toward treatment for individual patients that involves lifestyle changes as well as pharmacological therapy. These guidelines primarily recommend acetaminophen as first-line therapy for most patients. However, traditional nonselective and COX-2 selective NSAIDs are a mainstay in osteoarthritis management due to their well established efficacy. ${ }^{3-8}$ Topical analgesics, including topical NSAIDs, are recommended as an alternative therapeutic option in several guidelines, particularly for patients who are at risk for serious gastrointestinal or cardiovascular adverse events, such as elderly patients. ${ }^{3,5,6,17}$

In 2008, the UK-based National Institute for Clinical Excellence (NICE) published guidance recommending that topical NSAIDs, along with acetaminophen, should be the first pharmacological options in the management of osteoarthritis pain (after nonpharmacological treatment alternatives). NICE prioritizes the use of topical NSAIDs before the addition of oral nonselective or COX-2 selective NSAIDS, or opioid analgesics. The use of oral nonselective or COX-2 selective NSAIDs, starting at the lowest effective dosage and administered with gastroprotective agents regardless of the patient's risk for gastrointestinal adverse events, is recommended as a second-line treatment. ${ }^{8}$ The NICE guidelines highlight a move to consider topical NSAIDs as first-line therapy.

Diclofenac is one of the most frequently used NSAIDs in the treatment of osteoarthritis and is the active ingredient in each of the several topical NSAIDs approved for use in the US. Among the several approved topical diclofenac formulations, diclofenac sodium gel $1 \%$ and diclofenac sodium topical solution 1.5\% (TDiclo) are approved for the treatment of osteoarthritis. ${ }^{18,19}$ Diclofenac sodium gel is approved for the treatment of the pain of osteoarthritis in joints amenable to topical treatment, whereas TDiclo is approved for the treatment of the signs and symptoms of osteoarthritis of the knee. The purpose of this article is to review currently available data for the TDiclo formulation.

\section{Diclofenac sodium topical solution with DMSO}

Each $1 \mathrm{~mL}$ of TDiclo contains $16.05 \mathrm{mg}$ diclofenac sodium. TDiclo solution also contains $45.5 \%$ dimethyl sulfoxide (DMSO) vehicle, which can result in enhanced penetration of active drug through the skin..$^{20}$ The mechanism of action of DMSO is complex, including denaturation of proteins and interaction with the polar head groups of some lipid bilayers to loosen the packing geometry of the skin..$^{21,22}$ In addition, it may facilitate drug partitioning from the formulation into the solvent within the skin tissue. ${ }^{22}$

\section{Efficacy studies}

Five double-blind, randomized, clinical trials of TDiclo have been published (Table 1). ${ }^{23-27}$ Three trials were placebo-controlled and/or vehicle/active-controlled. ${ }^{23-25}$ One trial included a topical diclofenac arm, an oral diclofenac arm, a topical plus active control (oral diclofenac) arm, a placebo control, and a vehicle control, ${ }^{26}$ and one trial was an equivalence study comparing topical diclofenac with oral diclofenac. ${ }^{27}$

\section{Patient demographics}

All patients included in the five studies were adults with primary osteoarthritis of the knee based on standard radiological criteria (defined by joint space narrowing and marginal osteophytes in the medial, lateral, and patellofemoral compartments) ${ }^{28}$ The total number of patients in each study is shown in Table 1. The mean age of the included patients was approximately $60-65$ years, and approximately $60 \%-70 \%$ in each study were female. Patients applied TDiclo around the affected knee at a dose of 40 drops (about $1.2 \mathrm{~mL}$ ) four times daily ${ }^{23-26}$ or 50 drops (about $1.5 \mathrm{~mL}$ ) three times daily in the equivalence study. ${ }^{27}$ Concomitant NSAIDs and other analgesics were not allowed during the studies. Use of rescue acetaminophen was permitted in all studies except the equivalence study, ${ }^{27}$ with up to four $325 \mathrm{mg}$ tablets per day allowed in three studies, ${ }^{24-26}$ and up to two $325 \mathrm{mg}$ tablets four times daily allowed in the other study. ${ }^{23}$ Low-dose aspirin was allowed for cardiovascular prophylaxis in all studies.

In three of the five studies, patients were required to have experienced a flare of pain after washout of previous therapy (Table 1). Pain flares were defined as an increase on the Western Ontario and McMaster Universities (WOMAC) Osteoarthritis Index pain subscale of $\geq 2$ points and $25 \%$, score of $\geq 2$ (moderate) on at least one of the five questions of the pain subscale, and baseline total pain score $\geq 6 .{ }^{24}$ The WOMAC Osteoarthritis Index is a validated questionnaire consisting of 24 questions (five about pain, 17 about physical function, and two about stiffness).

\section{Outcome measures}

Efficacy variables in the studies included measures of pain, physical function, and patients' perception of their osteoarthritis symptoms and/or overall health (Table 1). It is important to note that this core set of outcome measures 


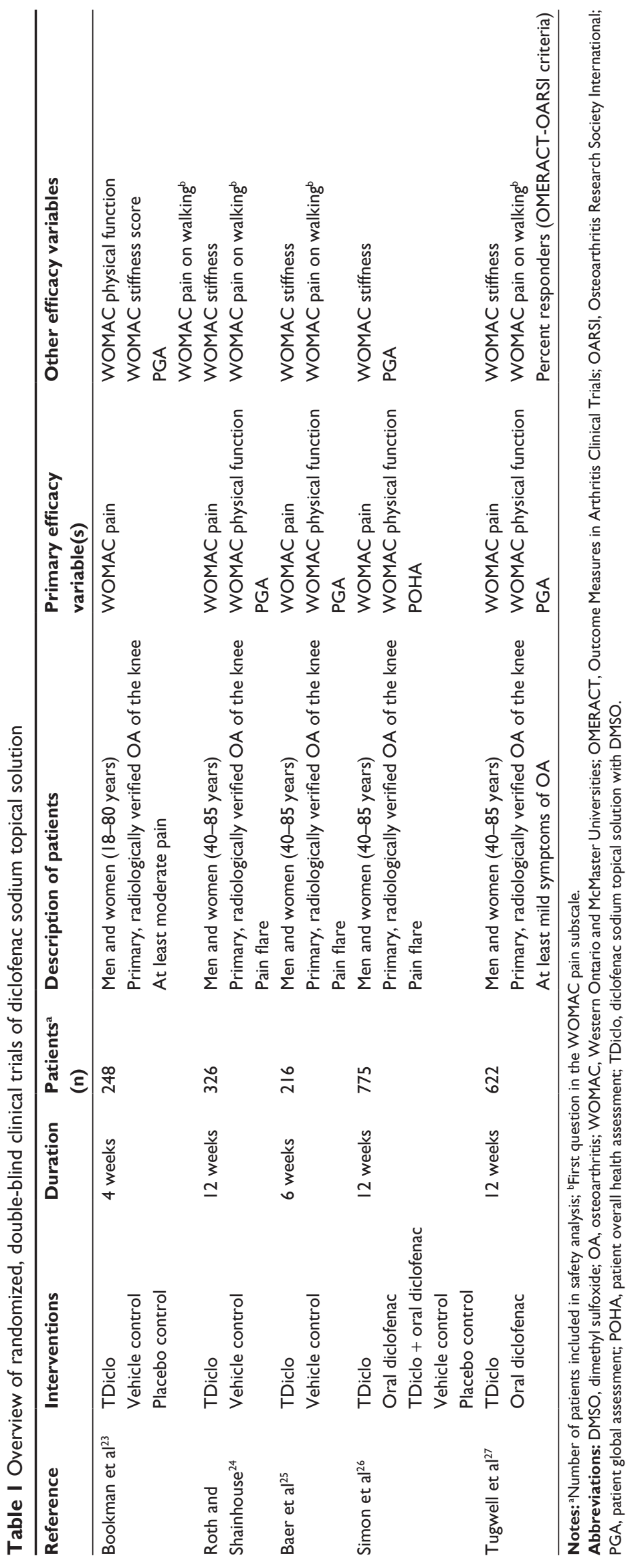


complies with the recommendations of the Outcome Measures in Arthritis Clinical Trials (OMERACT) III, ${ }^{29}$ the Osteoarthritis Research Society International (OARSI), ${ }^{30}$ and the Group for the Respect of Ethics and Excellence in Science. ${ }^{31}$ The WOMAC Osteoarthritis Index pain score, the WOMAC Osteoarthritis Index physical function score, and the Patient Global Assessment (PGA) score were coprimary efficacy variables in three of the five studies. One of the remaining studies utilized the Patient Overall Health Assessment (POHA) score instead of the PGA as the third coprimary endpoint. The PGA question asks patients about their osteoarthritis in the target knee during the past 48 hours, whereas the POHA question asks patients how they rated their overall state of health in the past 48 hours in relation to their osteoarthritis knee and its treatment. The fifth study evaluated only the WOMAC pain score as a primary endpoint and evaluated all other scales as a part of its secondary endpoints (Table 1). Secondary efficacy variables included the WOMAC stiffness subscale and pain on walking (the first question in the WOMAC pain assessment). All questions were scored on a five-point Likert scale, except in the equivalence study, in which questions were scored on a $100 \mathrm{~mm}$ visual analog scale.

\section{Efficacy vs DMSO vehicle or placebo}

In all four placebo-controlled or vehicle-controlled studies, TDiclo showed statistically significant improvements vs placebo and/or vehicle in WOMAC pain score (Figure 1). Mean improvement in scores for TDiclo vs vehicle control in each

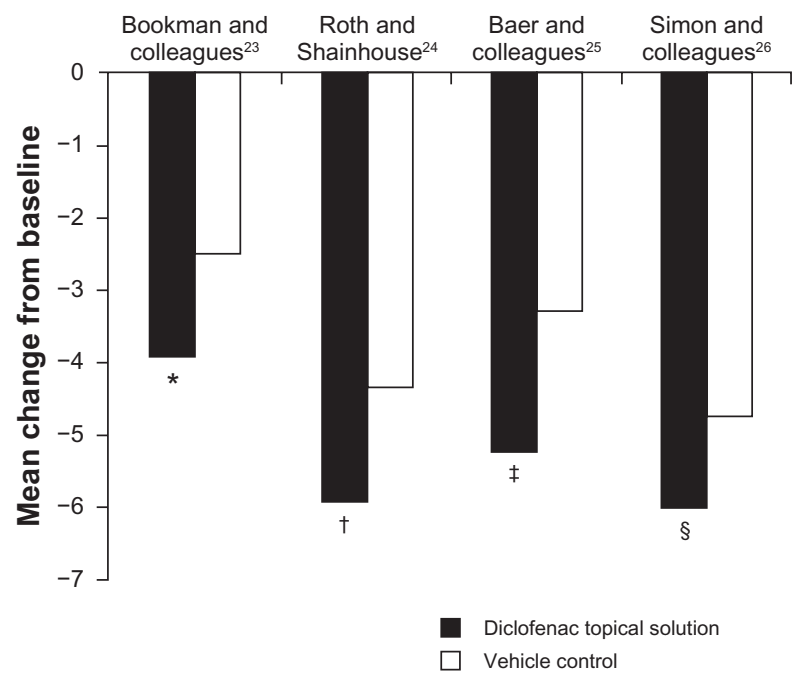

Figure I Improvement from baseline to final assessment in WOMAC pain score with diclofenac sodium topical solution and vehicle control. Pain score was based on five questions scored on a scale of 0 to 4 .

Notes: ${ }^{*} P=0.023 ;{ }^{\dagger} P=0.001 ; \ddagger P=0.003 ;{ }^{\$} P=0.009$. $P$ values are vs vehicle control. Abbreviation: WOMAC, Western Ontario and McMaster Universities. of the four studies was $-3.9 \mathrm{vs}-2.5(P=0.023),{ }^{23}-5.9 \mathrm{vs}-4.3$ $(P=0.001),{ }^{24}-5.2 \mathrm{vs}-3.3(P=0.003),{ }^{25}$ and $-6.0 \mathrm{vs}-4.7$ $(P=0.009) .{ }^{26}$ The mean percent improvement in WOMAC pain score from baseline to final assessment with TDiclo was $42.9 \%,{ }^{23} 45.7 \%,{ }^{24} 40.0 \%,{ }^{25}$ and $45.5 \% .{ }^{26}$ In contrast, the percent change with vehicle or placebo ranged between $26.0 \%$ and $36.4 \%$.

Statistically significant improvements in WOMAC physical function compared with vehicle and/or placebo were also shown in all four studies (Figure 2). Mean change in scores for TDiclo vs vehicle control in each of the four studies was -11.6 vs $-5.7(P=0.002),{ }^{23}-15.4$ vs $-10.1(P=0.002),{ }^{24}$ -13.4 vs $-6.9(P=0.001),{ }^{25}$ and -15.8 vs $-12.1(P=0.026){ }^{26}$ Improvements with TDiclo corresponded to a percent improvement from baseline to final assessment of $39.3 \%,{ }^{23}$ $36.7 \%,{ }^{24} 32.8 \%,{ }^{25}$ and $37.9 \%,{ }^{26}$ compared with $17.1 \%-29.6 \%$ for vehicle or placebo.

Patients who used TDiclo also reported better overall health status as assessed by the POHA or PGA, with a statistically significant difference vs vehicle and/or placebo in all studies (Table 2). ${ }^{23-26}$ Mean change from baseline to final assessment in PGA for TDiclo vs vehicle control in each of the three studies assessing PGA as a primary outcome was -1.3 vs $-0.9(P=0.003),{ }^{24}-1.3$ vs $-0.7(P=0.0001),{ }^{25}$ and -1.36 vs $-1.07(P=0.018) .{ }^{26}$ For TDiclo, the percent improvement was $42.2 \%,{ }^{24} 41.9 \%,{ }^{25}$ and $43.6 \%,{ }^{26}$ compared with $21.9 \%-34.2 \%$ for vehicle or placebo. Bookman et al assessed PGA as a secondary variable and showed similar results. ${ }^{23}$ After 4 weeks of treatment, TDiclo was associated

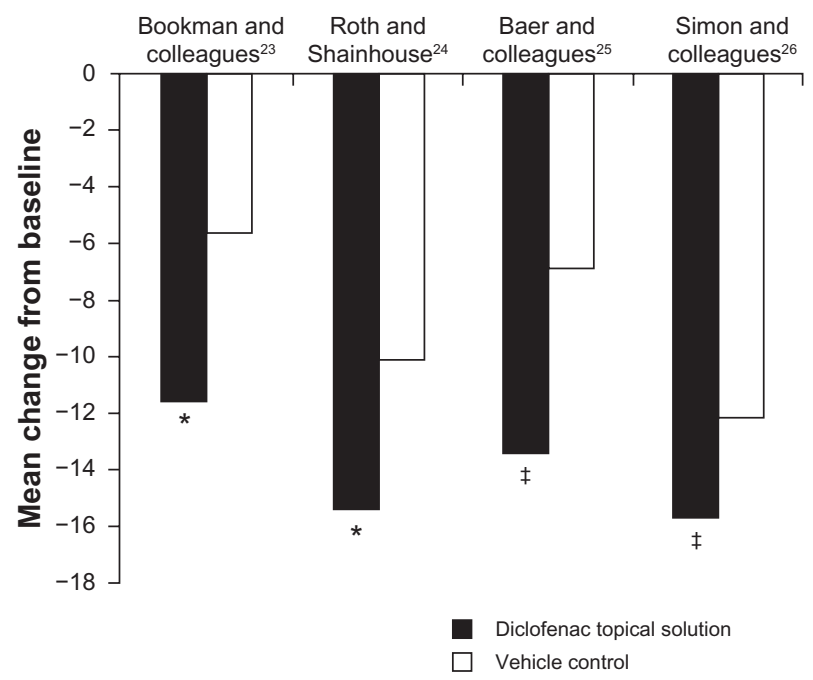

Figure 2 Improvement from baseline to final assessment in WOMAC physical function score with diclofenac sodium topical solution and vehicle control. Physical function score was based on 17 questions scored on a scale of 0 to 4 .

Notes: ${ }^{*} P=0.002 ;{ }^{\dagger} P=0.001 ;{ }^{\ddagger} P=0.026$. $P$ values are vs vehicle control. Abbreviation: WOMAC, Western Ontario and McMaster Universities. 
Table 2 Patient perception of osteoarthritis symptoms and overall health status as assessed by the PGA or POHA, after treatment with diclofenac sodium topical solution, vehicle control, or placebo

\begin{tabular}{|c|c|c|c|c|c|c|}
\hline Reference & Variable & TDiclo & $\begin{array}{l}\text { Vehicle } \\
\text { control }\end{array}$ & $P$ value $^{a}$ & $\begin{array}{l}\text { Placebo } \\
\text { control }\end{array}$ & $P$ value $^{\mathrm{b}}$ \\
\hline Bookman et $\mathrm{a}^{23}$ & $\begin{array}{l}\text { PGA (sum of scores at } \\
\text { weeks I, 2, 3, and 4) }\end{array}$ & 6.7 & 7.8 & 0.039 & 7.8 & 0.025 \\
\hline Roth and Shainhouse ${ }^{24}$ & PGA (change from baseline) & $-1.3(42.2 \%)$ & $-0.9(30.4 \%)$ & 0.003 & - & - \\
\hline Baer et $\mathrm{al}^{25}$ & PGA (change from baseline) & $-1.3(41.9 \%)$ & $-0.7(21.9 \%)$ & 0.0001 & - & - \\
\hline \multirow[t]{2}{*}{ Simon et $\mathrm{al}^{26}$} & PGA (change from baseline) & $-1.36(43.6 \%)$ & $-1.07(34.2 \%)$ & 0.018 & $-1.01(33.2 \%)$ & 0.016 \\
\hline & POHA (change from baseline) & $-0.95(40.6 \%)$ & $-0.65(28.3 \%)$ & 0.016 & $-0.37(16.7 \%)$ & $<0.000$ I \\
\hline
\end{tabular}

Notes: ${ }^{\mathrm{T} T D i c l o ~ v s ~ v e h i c l e ~ c o n t r o l ; ~}{ }^{\mathrm{b}}$ TDiclo vs placebo. PGA and POHA were assessed on a scale of 0 to 4 . Values shown indicate change from baseline (\% improvement). Abbreviations: OA, osteoarthritis; PGA, Patient Global Assessment; POHA, Patient Overall Health Assessment; TDiclo, diclofenac sodium topical solution.

with a significantly better mean PGA score (6.7) compared with vehicle control and placebo (7.8 in both groups, $P=0.039) .{ }^{23}$ A single study utilizing POHA showed a mean change from baseline to final assessment for TDiclo vs vehicle control of -0.95 vs $-0.65(P=0.016){ }^{26}$

Greater improvements in WOMAC stiffness score with TDiclo vs vehicle and/or placebo were also observed; all improvements were statistically significant $(P<0.05)$ other than the comparison with placebo in the study by Simon et al. ${ }^{26}$ Statistically significant $(P<0.05)$ improvements compared with vehicle and/or placebo were also seen for pain on walking. ${ }^{23-25}$

\section{Efficacy vs oral diclofenac}

Two studies of TDiclo have compared efficacy of the solution vs oral diclofenac. ${ }^{26,27}$ One of the studies also included placebo and vehicle control arms, as described above. ${ }^{26}$ In the oral diclofenac arm, patients received diclofenac $100 \mathrm{mg}$ once daily in a extended-release formulation (Novopharm Ltd, Stouffville, ON). The efficacy of TDiclo was equivalent to that of oral diclofenac, and no statistically significant differences were observed between the two treatments in a post hoc analysis. The mean changes in outcomes measurement scores for TDiclo vs oral diclofenac were -6.0 vs -6.4 for WOMAC pain score, -15.1 vs -17.5 for WOMAC physical function score, $-0.95 \mathrm{vs}-0.88$ for POHA, and -1.36 vs -1.42 for PGA. These differences were not statistically significant. Percent improvements for these outcome measures with TDiclo ranged from $38 \%$ to $45 \%$, and with oral diclofenac ranged from $39 \%$ to $48 \%$ (Figure 3). In addition to the oral diclofenac arm, this study also included a treatment arm in which patients received both oral diclofenac and the topical solution (Table 1). The combination did not produce greater improvements compared with oral diclofenac alone.

The second active comparator study was designed as an equivalence trial (Table 1). ${ }^{27}$ Oral diclofenac was administered at a dose of $50 \mathrm{mg}$ three times daily. The coprimary efficacy variables were WOMAC pain, WOMAC physical function, and PGA, measured on a $100 \mathrm{~mm}$ visual analog scale. To demonstrate equivalence between TDiclo and oral diclofenac, two-sided 95\% confidence intervals (CIs) were calculated for the difference between treatments in the mean change in score for each of the coprimary efficacy variables.
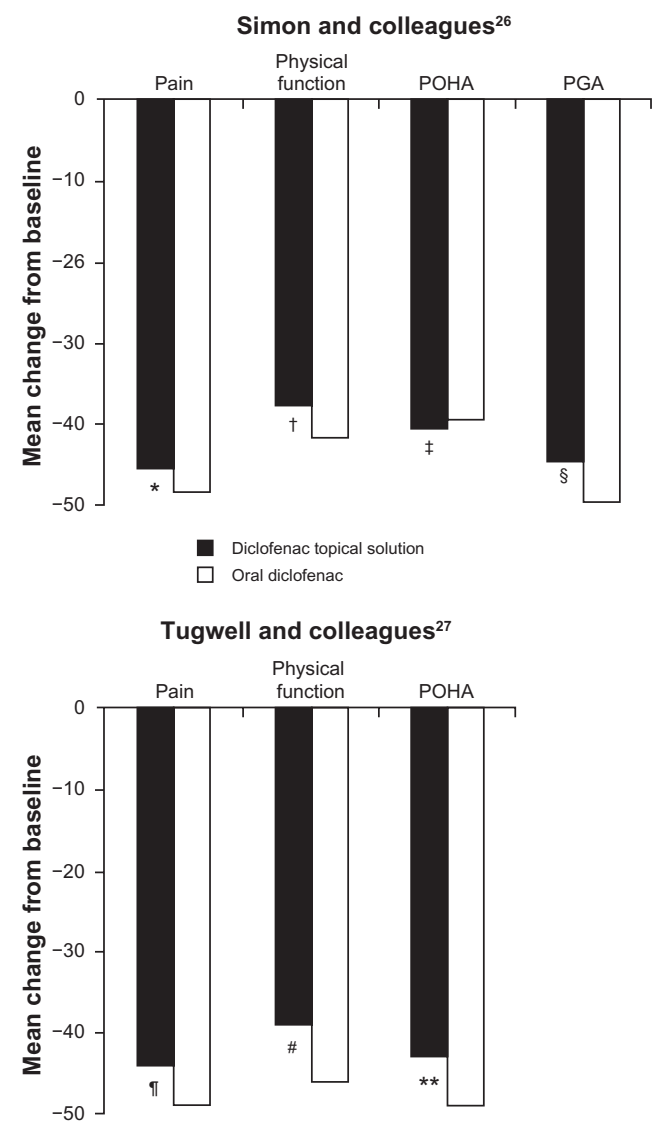

Figure 3 Percent change from baseline to final assessment in efficacy variables with diclofenac sodium topical solution and oral diclofenac.

Notes: $* P=0.429 ;{ }^{\dagger} P=0.319 ;{ }^{\sharp} P=0.956 ;{ }^{\S} P=0.439 ;{ }^{\top} P=0.23 ;{ }^{*} P=0.06 ; * * P=0.13$. $P$ values are vs oral diclofenac.

Abbreviations: PGA, Patient Global Assessment; POHA, Patient Overall Health Assessment. 
The treatments were considered equivalent if the CIs were in the range of -75 to $75 \mathrm{~mm}$ for WOMAC pain score $( \pm 15 \mathrm{~mm}$ for each of the five questions in the WOMAC pain subscale), -255 to $255 \mathrm{~mm}$ for WOMAC physical function score $( \pm 15 \mathrm{~mm}$ for each of the 17 questions in the WOMAC physical function subscale), and -20 to $20 \mathrm{~mm}$ for PGA. These limits were based on the minimal clinically important difference in osteoarthritis trials that was previously established by a panel of expert rheumatologists. ${ }^{32}$ The primary analysis was performed in the per protocol population, as is typical for equivalence studies.

The 95\% CIs for the three coprimary efficacy parameters were well within the predefined limits; therefore, the analysis demonstrated equivalence of TDiclo and oral diclofenac. The difference between treatments in mean change in score from baseline was $13.3 \mathrm{~mm}(95 \% \mathrm{CI}$ : -8.6-35.2) for WOMAC pain score, $71.0 \mathrm{~mm}$ (95\% CI: -2.4-144.5) for WOMAC physical function score, and $4.3 \mathrm{~mm}(95 \% \mathrm{CI}$ : -1.2-9.8) for PGA. TDiclo treatment resulted in a 44\% improvement in pain, 39\% improvement in physical function, and 43\% improvement in PGA (Figure 3). ${ }^{26,27}$ No statistically significant differences were seen between treatments in the per protocol data set. Similar efficacy results were seen for stiffness and pain on walking.

\section{Safety data}

\section{Plasma levels of diclofenac}

The concentration of diclofenac reached in plasma is considerably lower after topical application compared with oral administration. In a study of a single application of $1 \mathrm{~mL}$ TDiclo to one knee in healthy volunteers, the maximum plasma concentration was $11.8 \pm 4.2 \mathrm{ng} / \mathrm{mL}$ (standard deviation) after $24-48$ hours. ${ }^{33}$ In another single-dose study in healthy volunteers, the maximum plasma concentration was $8.1 \pm 5.9 \mathrm{ng} / \mathrm{mL}$ approximately 10 hours after an application of 40 drops (approximately $1.2 \mathrm{~mL}$ ) to each knee. ${ }^{19}$ Following application of 40 drops to each knee four times daily for 7 days in healthy volunteers, maximum plasma concentration at steady state was $19.4 \pm 9.3 \mathrm{ng} / \mathrm{mL} .{ }^{19}$ In contrast, following administration of oral diclofenac $50 \mathrm{mg}$ every 8 hours in a separate study, maximum plasma concentration at steady state was $2270 \mathrm{ng} / \mathrm{mL}{ }^{34}$

\section{Tolerability and adverse events vs oral diclofenac}

The incidence of adverse events occurring in the two randomized, controlled studies comparing TDiclo with oral diclofenac is shown in Table 3. ${ }^{26,27}$ Both studies included a dermatological assessment of the knee to evaluate application site reactions, ${ }^{26,27}$ and the equivalence study included a statistical comparison of adverse event incidence between the study groups. ${ }^{27}$ Most adverse events occurring in patients treated with TDiclo were application site reactions. Dry skin was by far the most common, occurring in $27 \%$ of patients treated with TDiclo in the equivalence study ${ }^{27}$ and in $18.2 \%$ of patients in the other active controlled study ${ }^{26}$ (Table 3 ). In the equivalence study, a statistically significant higher incidence of dry skin, rash, pruritus, and vesiculobullous rash occurred with TDiclo compared with oral diclofenac (Table 3). ${ }^{27}$ More patients withdrew due to skin-related adverse events in the TDiclo group than in the oral diclofenac group (10\% vs $0.3 \%$, $P<0.0001) .{ }^{27}$ A much lower rate of study discontinuation due to application site reactions $(3.2 \%)$ was seen in the TDiclo arm of the other active-controlled study. ${ }^{26}$ The dry skin seems to be in part related to the DMSO vehicle, because it was also observed in patients treated with vehicle control. ${ }^{23-26}$ In clinical practice, emollients may be used after the topical solution has dried in order to alleviate skin dryness; however, this practice was not specifically evaluated during the course

Table 3 Adverse events in studies comparing diclofenac sodium topical solution with oral diclofenac ${ }^{26,27}$

\begin{tabular}{|c|c|c|c|c|}
\hline \multirow{2}{*}{$\begin{array}{l}\text { Adverse events } \\
\text { (\%) }\end{array}$} & \multicolumn{2}{|c|}{ Tugwell et $\mathrm{al}^{27}$} & \multicolumn{2}{|c|}{ Simon et $\mathrm{al}^{26}$} \\
\hline & TDiclo & $\begin{array}{l}\text { Oral } \\
\text { diclofenac }\end{array}$ & TDiclo & $\begin{array}{l}\text { Oral } \\
\text { diclofenac }\end{array}$ \\
\hline \multicolumn{5}{|l|}{ Application site } \\
\hline Dry skin & 27 & $I^{a}$ & 18.2 & 2.6 \\
\hline Rash & 12 & $2^{\mathrm{a}}$ & - & - \\
\hline Paresthesia & 0.6 & 0.6 & - & - \\
\hline Pruritus & 6 & $0.6^{\mathrm{a}}$ & 1.3 & 0.0 \\
\hline Urticaria & 0.3 & 0.3 & - & - \\
\hline Vesiculobullous rash & 5 & $0^{\mathrm{a}}$ & 1.9 & 0.7 \\
\hline Contact dermatitis & - & - & 2.6 & 0.7 \\
\hline \multicolumn{5}{|l|}{ Gastrointestinal } \\
\hline All Gl events & 35 & $48^{\mathrm{a}}$ & 6.5 & 23.8 \\
\hline Abdominal pain & 12 & $22^{\mathrm{a}}$ & 3.2 & 7.3 \\
\hline Constipation & 8 & 10 & - & - \\
\hline Diarrhea & 9 & $17^{\mathrm{a}}$ & 1.3 & 4.6 \\
\hline Dyspepsia & 15 & $26^{\mathrm{a}}$ & 2.6 & 4.0 \\
\hline Flatulence & 10 & $17^{\mathrm{a}}$ & - & - \\
\hline Melena & I & 2 & - & - \\
\hline Nausea & 8 & $13^{\mathrm{a}}$ & 0.0 & 2.0 \\
\hline Vomiting & 2 & 2 & ND & ND \\
\hline \multicolumn{5}{|c|}{ Laboratory parameters ${ }^{b}$} \\
\hline AST & 2 & $10^{\mathrm{a}}$ & 6.9 & 19.6 \\
\hline ALT & 5 & $17^{\mathrm{a}}$ & 4.1 & 18.8 \\
\hline Hemoglobin & 2 & $10^{\mathrm{a}}$ & 2.1 & 5.8 \\
\hline Creatinine & I & 3 & 2.8 & 7.2 \\
\hline
\end{tabular}

Notes: ${ }^{\text {a }}>0.05$ vs TDiclo with DMSO. No statistical analysis was performed in Simon et al; ${ }^{26}$ bercentage of patients changing from normal levels at baseline to abnormal levels during the study.

Abbreviations: GI, gastrointestinal; AST, aspartate aminotransferase; ALT, alanine aminotransferase; DMSO, dimethyl sulfoxide; TDiclo, diclofenac sodium topical solution. 
of the study, so it is not known to what extent emollients will alleviate these application site reactions.

Gastrointestinal adverse events occurred more frequently with oral diclofenac in both active-controlled studies (Table 3 ). In the study reported by Simon et al, ${ }^{26}$ gastrointestinal adverse events occurred in $23.8 \%$ of patients receiving oral diclofenac vs $6.5 \%$ of patients receiving TDiclo. In the equivalence study, gastrointestinal events occurred in $48 \%$ of patients receiving oral diclofenac vs $35 \%$ of patients receiving TDiclo $(P<0.05)$. Specific gastrointestinal adverse events for which a statistically significant difference between groups was shown included abdominal pain, diarrhea, dyspepsia, flatulence, and nausea (Table 3). There was no significant difference between the study groups with respect to constipation, melena, and vomiting. The proportion of patients with a gastrointestinal adverse event classified as severe was greater in the oral diclofenac group $(21.3 \%)$ compared with the TDiclo group (7.4\%), and the same pattern was observed for abdominal pain, dyspepsia, and diarrhea. ${ }^{27}$

Significantly more patients withdrew due to gastrointestinal adverse events in the oral diclofenac group than in the TDiclogroup ( $16 \%$ vs $6 \%$, respectively, $P<0.0001$ ). Interestingly, a much lower incidence of gastrointestinal adverse events was reported with TDiclo in the three randomized, placeboand/or vehicle-controlled studies ${ }^{23-25}$ compared with the active-controlled studies. This is consistent with a systematic review of oral NSAID trials that showed a higher incidence of gastrointestinal adverse events in active comparator trials compared with placebo-controlled trials. ${ }^{35}$

In addition, laboratory parameters changed from normal to abnormal levels in a greater number of patients receiving oral diclofenac compared with TDiclo in both active-controlled studies (Table 3). In the equivalence study, a statistically significant difference between treatment groups was observed in elevations in aspartate aminotransferase, alanine aminotransferase, and hemoglobin levels. However, there was no difference between study groups in the incidence of hypertension or cardiovascular events in either study. ${ }^{26,27}$

\section{Long-term safety}

TDiclo was well tolerated in controlled clinical trials of 6-12 weeks' duration. However, because patients with osteoarthritis of the knee are likely to use topical NSAIDs over the course of many years, it is important to gain an understanding of long-term safety reflecting use in clinical practice. An open-label, prospective study evaluated the safety of TDiclo for up to 52 weeks. ${ }^{36}$ The study was conducted at outpatient centers and included 793 patients aged 35-85 (mean 62.5) years with radiologically confirmed osteoarthritis of the knee. Patients applied 40 drops of TDiclo to the knee four times daily. The most common adverse event reported was dry skin at the application site $(25.3 \%$ of patients), followed by contact dermatitis (13.0\%) and contact dermatitis with vesicles $(9.5 \%)$. However, dermatological assessment of the application site showed that most patients had a normal skin irritation score (Figure 4). ${ }^{36}$

In this open-label, long-term study, gastrointestinal adverse events occurred in $12 \%$ of patients, including abdominal pain in $2.3 \%$, gastroesophageal reflux in $1.6 \%$, diarrhea in $1.4 \%$, dyspepsia in $1.4 \%$, and nausea in $1.1 \%$. A total of 28 cases of hypertension were reported $3.5 \%$ of patients). ${ }^{36}$ However, most of these patients had hypertension at study enrolment; only $13(1.6 \%)$ were considered to be true cases of new-onset or worsening hypertension. Other cardiovascular adverse events that occurred in $\geq 0.5 \%$ of patients were peripheral edema $(1.4 \%)$, angina/chest pain $(0.8 \%)$, palpitation $(0.6 \%)$, myocardial infarction $(0.5 \%)$, arrhythmia $(0.5 \%)$, deep vein thrombosis $(0.5 \%)$, and vasodilatation $(0.5 \%)$. Prolonged exposure to TDiclo with DMSO resulted in few changes in hemoglobin, aspartate aminotransferase, alanine aminotransferase, or creatinine values. A change from normal to abnormal levels occurred in $3.2 \%$ of patients for hemoglobin, $6.4 \%$ for aspartate aminotransferase, $7.3 \%$ for alanine aminotransferase, and $4.2 \%$ for creatinine. ${ }^{36}$

\section{DMSO-related adverse reactions}

Preclinical studies of DMSO have resulted in rare reports of ocular lens abnormalities with high-dose exposure in nonprimate species, ${ }^{37}$ although studies in humans have found

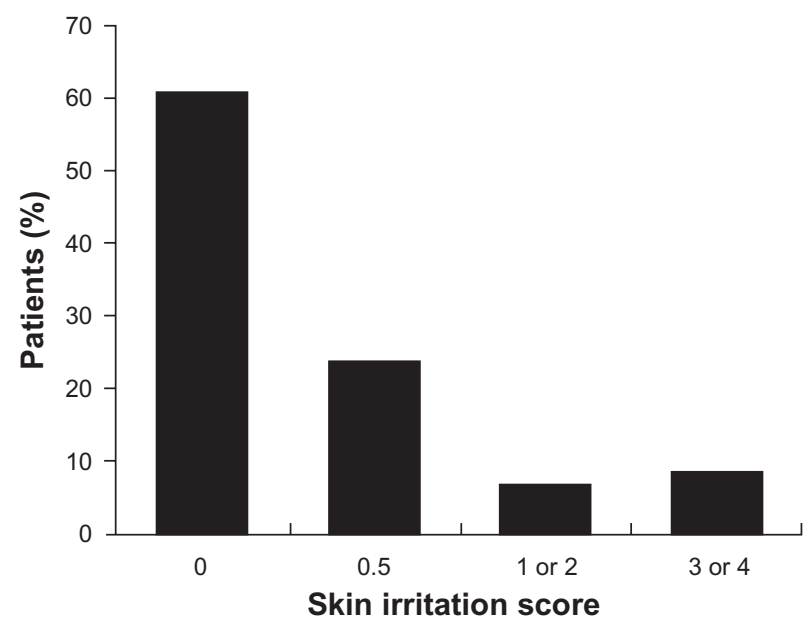

Figure 4 Skin irritation scores in a long-term, open-label study of diclofenac sodium topical solution. ${ }^{36}$

Notes: $0=$ normal; $0.5=$ dryness or flaking; I or $2=$ erythema with or without induration; 3 or $4=$ erythema with induration and vesicles or bullae. 
no ocular toxicity even with high doses applied topically or directly to the eye. ${ }^{38-40}$ In the controlled trial reported by Simon et $\mathrm{al},{ }^{26}$ there was no increase in eye abnormalities in the TDiclo groups or the vehicle control group compared with placebo. In the long-term safety study, cataracts were reported in 18 patients (3\% of those who received an ocular examination); seven patients developed a new cataract, whereas progression of an existing cataract was reported in 11 patients. ${ }^{36}$ These cataract event rates appeared to be somewhat lower than seen in natural history studies. ${ }^{41-45}$ Another adverse reaction that may be attributed to DMSO is a garlic taste or odor, due to exhalation of dimethyl sulfide, a metabolite of DMSO. However, a low incidence of taste perversion $(0.6 \%-3.7 \%)$ and halitosis $(0 \%-5 \%)$ was reported in all studies reported here. . $3-27,36^{2}$

\section{Comments and conclusion}

Oral nonselective NSAIDs have shown to be effective and are widely used in the treatment of osteoarthritis, but these agents are associated with a risk of serious gastrointestinal and cardiovascular adverse events. ${ }^{9}$ These events, which can be fatal and may occur without warning, are the basis of the boxed warning in the prescribing information required by the FDA and the required risk evaluation and mitigation strategy program, which includes a medication guide. ${ }^{9,46}$ When determining a pain management plan in patients with osteoarthritis, minimizing risk is a priority. ${ }^{9}$

TDiclo may provide analgesia similar to oral NSAIDs with significantly lower dosage and systemic absorption of the active drug. Lower systemic concentrations may therefore result in less risk of systemic adverse events, including the gastrointestinal events discussed above. At this time, the prescribing information for topical NSAIDs also includes the classwide boxed warning regarding gastrointestinal and cardiovascular events. ${ }^{18,19}$ Based on the summary of data reported here, by adopting topical NSAID treatment as the first method of intervention for osteoarthritis, healthcare professionals may be able to decrease the risk of adverse events without compromising the efficacy of treatment.

TDiclo demonstrated efficacy in osteoarthritis of the knee similar to oral diclofenac in large, well-designed, randomized, controlled trials. Dry skin was the most frequent adverse event observed, but systemic NSAID class-related adverse events and laboratory abnormalities were considerably less with TDiclo compared with oral diclofenac. Topical NSAIDs have not been included in some clinical guideline recommendations because of a lack of data concerning overall efficacy.
As more data about topical NSAIDs are published, health organizations will have new opportunities to evaluate these data as they update their existing recommendations. Clinical studies to date show that TDiclo represents a practical and evidence-based option for the management of osteoarthritis of the knee.

\section{Disclosure}

$\mathrm{PF}$ is an employee of Covidien, the distributor of Pennsaid ${ }^{\circledR}$ (diclofenac sodium topical solution $1.5 \% \mathrm{w} / \mathrm{w}$ ) in the US. SR serves as a consultant and speaker for Covidien, and is a current stakeholder in Transdel Pharmaceuticals.

\section{References}

1. Lawrence RC, Felson DT, Helmick CG, et al. National Arthritis Data Workgroup. Estimates of the prevalence of arthritis and other rheumatic conditions in the United States. Part II. Arthritis Rheum. 2008; 58(1):26-35.

2. Bitton R. The economic burden of osteoarthritis. Am J Manag Care. 2009;15(Suppl 8):S230-S235.

3. American College of Rheumatology Subcommittee on Osteoarthritis Guidelines. Recommendations for the medical management of osteoarthritis of the hip and knee: 2000 update. Arthritis Rheum. 2000;43(9):1905-1915.

4. Jordan KM, Arden NK, Doherty M, et al. Standing Committee for International Clinical Studies Including Therapeutic Trials ESCISIT. EULAR Recommendations 2003: an evidence based approach to the management of knee osteoarthritis: report of a Task Force of the Standing Committee for International Clinical Studies Including Therapeutic Trials (ESCISIT). Ann Rheum Dis. 2003;62(12):1145-1155.

5. Zhang W, Moskowitz RW, Nuki G, et al. OARSI recommendations for the management of hip and knee osteoarthritis, Part II: OARSI evidence-based, expert consensus guidelines. Osteoarthritis Cartilage. 2008;16(2):137-162.

6. Tannenbaum H, Bombardier C, Davis P, Russell AS. Third Canadian Consensus Conference Group. An evidence-based approach to prescribing nonsteroidal antiinflammatory drugs. Third Canadian Consensus Conference. J Rheumatol. 2006;33(1):140-157.

7. Chou R, Helfand M, Peterson K, Dana T, Roberts C. Comparative Effectiveness and Safety of Analgesics for Osteoarthritis. Comparative Effectiveness Review No. 4. Rockville, MD: agency for Healthcare Research and Quality; September 2006. Available from: http://www. effectivehealthcare.ahrq.gov/reports/final.cfm. Accessed May 5, 2010.

8. National Collaborating Centre for Chronic Conditions. Osteoarthritis: National Clinical Guideline for Care and Management in Adults. London, UK: Royal College of Physicians; 2008. Available from: http://www.nice. org.uk/nicemedia/pdf/cg059fullguideline.pdf. Accessed May 5, 2010.

9. Roth SH. Nonsteroidal antiinflammatory drug gastropathy: we started it, why don't we stop it? J Rheum. 2005;32(7):1189-1191.

10. Antman EM, Bennett JS, Daugherty A, Furberg C, Roberts H, Taubert KA. American Heart Association. Use of nonsteroidal antiinflammatory drugs: an update for clinicians: a scientific statement from the American Heart Association. Circulation. 2007;115(12):1634-1642.

11. Hippisley-Cox J, Coupland C, Logan R. Risk of adverse gastrointestinal outcomes in patients taking cyclo-oxygenase-2 inhibitors or conventional non-steroidal anti-inflammatory drugs: population based nested case-control analysis. BMJ. 2005;331(7528):1310-1316.

12. Kearney PM, Baigent C, Godwin J, Halls H, Emberson JR, Patrono C. Do selective cyclo-oxygenase-2 inhibitors and traditional non-steroidal anti-inflammatory drugs increase the risk of atherothrombosis? Metaanalysis of randomised trials. BMJ. 2006;332(7553):1302-1308. 
13. Mamdani M, Juurlink DN, Kopp A, Naglie G, Austin PC, Laupacis A. Gastrointestinal bleeding after the introduction of COX 2 inhibitors: ecological study. BMJ. 2004;328(7453):1415-1416.

14. Moore RA, Derry S, McQuay HJ. Cyclo-oxygenase-2 selective inhibitors and nonsteroidal anti-inflammatory drugs: balancing gastrointestinal and cardiovascular risk. BMC Musculoskelet Disord. 2007;8:73

15. Harirforoosh S, Jamali F. Renal adverse effects of nonsteroidal antiinflammatory drugs. Expert Opin Drug Saf. 2009;8(6):669-681.

16. Moore RA, Derry S, McQuay HJ. Topical agents in the treatment of rheumatic pain. Rheum Dis Clin North Am. 2008;34(2):415-432.

17. American Geriatrics Society Panel on the Pharmacological Management of Persistent Pain in Older Persons. Pharmacological management of persistent pain in older persons. J Am Geriatric Soc. 2009;57(8): 1331-1346.

18. Voltaren Gel (diclofenac sodium topical gel) 1\% Prescribing information. Chadds Ford, PA: Endo Pharmaceuticals Inc; 2009.

19. Pennsaid (diclofenac sodium topical solution) $1.5 \% \mathrm{w} / \mathrm{w}$ Prescribing information. Hazelwood, MO: Mallinckrodt Inc; 2010.

20. Hewitt PG, Poblete N, Wester RC, Maibach HI, Shainhouse JZ. In vitro cutaneous disposition of a topical diclofenac lotion in human skin: effect of a multi-dose regimen. Pharm Res. 1998;15(7):988-992.

21. Wiechers JW. The barrier function of the skin in relation to percutaneous absorption of drugs. Pharm Weekbl Sci. 1989;11(6):185-198.

22. Williams AC, Barry BW. Penetration enhancers. Adv Drug Deliv Rev. 2004;56(5):603-618.

23. Bookman AA, Williams KS, Shainhouse JZ. Effect of a topical diclofenac solution for relieving symptoms of primary osteoarthritis of the knee: a randomized controlled trial. CMAJ. 2004;171(4):333-338.

24. Roth SH, Shainhouse JZ. Efficacy and safety of a topical diclofenac solution (Pennsaid) in the treatment of primary osteoarthritis of the knee: a randomized, double-blind, vehicle-controlled clinical trial. Arch Intern Med. 2004;164(18):2017-2023.

25. Baer PA, Thomas LM, Shainhouse Z. Treatment of osteoarthritis of the knee with a topical diclofenac solution: a randomised controlled, 6-week trial [ISRCTN53366886]. BMC Musculoskelet Disord. 2005;6:44.

26. Simon LS, Grierson LM, Naseer Z, Bookman AA, Zev Shainhouse J. Efficacy and safety of topical diclofenac containing dimethyl sulfoxide (DMSO) compared with those of topical placebo, DMSO vehicle and oral diclofenac for knee osteoarthritis. Pain. 2009;143(3):238-245.

27. Tugwell PS, Wells GA, Shainhouse JZ. Equivalence study of a topical diclofenac solution $\left(\right.$ Pennsaid $^{\mathbb{1}}$ ) compared with oral diclofenac in symptomatic treatment of osteoarthritis of the knee: a randomized controlled trial. J Rheumatol. 2004;31(10):2002-2012.

28. Altman RD, Hochberg M, Murphy WAJ, Wolfe E, Lequesne M. Atlas of individual radiographic features in osteoarthritis. Osteoarthritis Cartilage. 1995;(3Suppl A):3-70.

29. Bellamy N, Kirwan J, Boers M, et al. Recommendations for a core set of outcome measures for future phase III clinical trials in knee, hip, and hand osteoarthritis. Consensus development at OMERACT III. J Rheumatol. 1997;24(4):799-802.

30. Altman R, Brandt K, Hochberg M, et al. Design and conduct of clinical trials in patients with osteoarthritis: recommendations from a task force of the Osteoarthritis Research Society. Results from a workshop. Osteoarthritis Cartilage. 1996;4(4):217-243.
31. Group for the Respect of Ethics and Excellence in Science (GREES): osteoarthritis section. Recommendations for the registration of drugs used in the treatment of osteoarthritis. Ann Rheum Dis. 1996;55(8): $552-557$.

32. Bellamy N, Carette S, Ford PM, et al. Osteoarthritis antirheumatic drug trials. III. Setting the delta for clinical trials - results of a consensus development (Delphi) exercise. J Rheumatol. 1992;19(3):451-457.

33. Hui X, Hewitt PG, Poblete N, Maibach HI, Shainhouse JZ, Wester RC. In vivo bioavailability and metabolism of topical diclofenac lotion in human volunteers. Pharm Res. 1998;15(10):1589-1595.

34. Kienzler JL, Gold M, Nollevaux F. Systemic bioavailability of topical diclofenac sodium gel 1\% versus oral diclofenac sodium in healthy volunteers. J Clin Pharmacol. 2010;50(1):50-61.

35. Rochon PA, Binns MA, Litner JA, et al. Are randomized control trial outcomes influenced by the inclusion of a placebo group? A systematic review of nonsteroidal antiinflammatory drug trials for arthritis treatment. J Clin Epidemiol. 1999;52(2):113-122.

36. Shainhouse JZ, Grierson LM, Naseer Z. A long-term, open-label study to confirm the safety of topical diclofenac solution containing dimethyl sulfoxide in the treatment of the osteoarthritic knee. Am J Ther. 2010; 17(6):566-576.

37. Rubin LF. Toxicologic update of dimethyl sulfoxide. Ann N Y Acad Sci. 1983;411:6-10.

38. Brobyn RD. The human toxicology of dimethyl sulfoxide. Ann NY Acad Sci. 1975;243:497-506.

39. Garcia CA. Ocular toxicology of dimethyl sulfoxide and effects on retinitis pigmentosa. Ann NY Acad Sci. 1983;411:48-51.

40. Shirley HH, Lundergan MK, Williams HJ, Spruance SL. Lack of ocular changes with dimethyl sulfoxide therapy of scleroderma. Pharmacotherapy. 1989;9(3):165-168.

41. Taylor HR, Muñoz B. The incidence and progression of lens opacities. Aust N Z J Ophthalmol. 1991;19(4):353-356.

42. Italian-American Cataract Study Group. Incidence and progression of cortical, nuclear, and posterior subcapsular cataracts. Am JOphthalmol. 1994;118(5):623-631.

43. Leske MC, Chylack LT Jr, He Q, et al. Incidence and progression of cortical and posterior subcapsular opacities: the Longitudinal Study of Cataract. The LSC Group. Ophthalmology. 1997;104(12): 1987-1993.

44. Leske MC, Chylack LT Jr, Wu SY, et al. Incidence and progression of nuclear opacities in the Longitudinal Study of Cataract. Ophthalmology. 1996;103(5):705-712.

45. Klein BE, Klein R, Lee KE. Incidence of age-related cataract: the Beaver Dam Eye Study. Arch Ophthalmol. 1998;116(2):219-225.

46. US Food and Drug Administration. Public Health Advisory - FDA announces important changes and additional warnings for COX-2 selective and non-selective non-steroidal anti-inflammatory drugs (NSAIDs) Available from: http://www.fda.gov/Drugs/DrugSafety/Postmarket DrugSafetyInformationforPatientsandProviders/ucm $150314 . \mathrm{htm}$. Accessed August 11, 2010.
Journal of Multidisciplinary Healthcare

\section{Publish your work in this journal}

The Journal of Multidisciplinary Healthcare is an international, peerreviewed open-access journal that aims to represent and publish research in healthcare areas delivered by practitioners of different disciplines. This includes studies and reviews conducted by multidisciplinary teams as well as research which evaluates the results or conduct of such teams or

\section{Dovepress}

healthcare processes in general. The journal covers a wide range of areas and welcomes submission from practitioners at all levels, from all over the world. The manuscript management system is completely online and includes a very quick and fair peer-review system. Visit http://www.dovepress.com/testimonials.php to read real quotes from published authors. 